\title{
$\angle$ Community practice in the time of COVID-19
}

\author{
J. Adair Prall, MD, ${ }^{1}$ John D. Davis, MD, MHS, ${ }^{2}$ and N. Ross Jenkins, MD $^{3}$ \\ ${ }^{1}$ NeurosurgeryOne, Denver, Colorado; ${ }^{2}$ NewSouth NeuroSpine, Jackson, Mississippi; and ${ }^{3}$ New Hampshire Neurospine Institute, \\ Concord, New Hampshire
}

'I 'M sorry but your case tomorrow morning is canceled. We don't have any N95s and don't know where we can get any." Such is the news on any given day in the last several weeks at our surgery center. Although it would be tempting to use terms like "unprecedented," "unheard of," and "apocalyptic," these words have largely lost their impact in a time with 10-minute news cycles and exponential daily changes in case and death counts from the novel COVID-19 coronavirus. Perhaps the most interesting aspect of the spread of this disease and its impact is the sheer unpredictability of its appearance and progression in different places. Near the end of March, three of us in community practice had a chance to compare our experiences thus far during the 2020 COVID-19 outbreak. In the following paragraphs, we have tried to paint a picture of how our own practices have been impacted and have adapted to our new, hopefully transient, reality.

The last 3 weeks have been extremely disorienting. When in the past have neurosurgeons had so many decisions made for them and been required to limit, change, or stop altogether their workflow? For a group of people who are not used to being told what we can and cannot do regarding basic patient care and medical decision-making, especially by administrators and other physicians, and more recently, local, state, and national politicians, this is a strange time for independent private practitioners. Those physicians employed by institutions such as hospitals and universities may have lived to some degree with this experience for some time, but it is quite unusual for those of us in the private setting. From calls to limit surgical cases to "urgent or emergent cases only," to patients deciding whether it is safe in your medical office building or not, sometimes insisting on telemedical appointments when in-person wound checks and follow-up clinical exams are indicated, it is easy to feel a loss of control in managing our practices and daily routines.
Around the country, there are many changes that have become ubiquitous. Frequent hand-washing; screening of patients for flu symptoms; limiting visitors and companions in medical offices, hospitals, and ambulatory surgery centers (ASCs); and constraints on surgical cases are being seen everywhere. The onset of these new practices has been slightly different in different locations, but they have arrived eventually to us all. Differences in degrees of screening, whether at the door of the office building or in the surgery scheduling office, can be significant. With surgical case screening, even in different hospitals within the same health system, there can be more or less scrutiny about what constitutes "necessary/urgent surgery." Of course the definition of this treatment category may differ in every practice, and therefore often boils down to whether the members of the oversight committee for surgical case screening are more conservative or liberal. Another constraint in some areas is the recent policy of some malpractice carriers to threaten not to provide liability coverage for clearly elective procedures, though this has not actually happened yet.

Although the extremes of the spectrum of urgency in surgical cases are obvious, a carpal tunnel release versus an acute subdural hematoma with midline shift, the majority of our patients are in the middle group between these two extremes. In most community practices, degenerative spine cases comprise the majority of the workload, and sometimes these procedures can be easily postponed; however, the majority of these cases are in this large "middle group," who are often patients with weakness or other functional deficits. We have had to become advocates for our patients in a way usually reserved for conversations with insurers, in many cases pushing our hospital and ASC policymakers to accept that many of our spine cases are urgent and cannot be easily or safely delayed. With this new process has come the additional mandate for documentation of "urgency." 
ASCs have become, in some areas, a pressure-relief valve for cases that need to be done and have been delayed in hospitals, or for patients with anxiety about exposure in large medical facilities. In other places, however, the ASCs have had more difficulty obtaining masks and other personal protective equipment (PPE) than the hospitals. In both settings, there seem to be major differences in the anesthesia providers' desire for more extensive PPE, especially during intubation. This use of more extensive PPE may be more of a strategic decision on the part of certain groups, to keep more providers working and avoid exposure and the resultant downtime with quarantine, yet the additional drain on limited PPE has made limitations on surgeries that much more stringent until new supplies eventually arrive.

In addition to the decreases in scheduled surgeries, we have noticed decreased emergency room (ER) and inpatient volumes as well, resulting in very low neurosurgical censuses in many areas. Speculation about the causes for quieter ERs (other than COVID-19 rule-outs) focuses around the idea that fewer people are outside their homes to injure themselves, whether driving while impaired, falling and suffering compression fractures, or being active enough to cause the flare up of a back or neck condition that requires a visit for acute pain management.

In the office, practices are largely turning to telehealth to maintain their clinic volumes, albeit with decreases of $50 \%$ or more, despite these efforts. This reliance on telemedicine seems to depend largely on the generational distribution of the patient referral base and their associated comfort level with remote communication tools like Zoom or Webex. One practice has relied more heavily on telephone consultations, simply offering an opening to begin dialogue with a neurosurgeon about a set of symptoms. With changes in billing requirements, remote consultations have served to keep patients coming into practices. Patients are generally expressing appreciation of the telehealth option, which limits possible exposure as well as extra office visits. Many are wondering if community practices might not be the beginning of a new mode of triage even after this episode ends.
Managing the sudden and artificially decreased volumes of our practices has been a great challenge. There is hope that the small business provisions of the recently passed Coronavirus Aid, Relief, and Economic Security (CARES) Act will provide some financial relief, though the practical details are only now being slowly unveiled. Unlike businesses in other sectors who have let most of their employees go and hope to win them back later, for medical providers the labor market for good staff has been so competitive leading up to this moment that our practices, as well as hospital systems and ASCs, are reluctant to let any employees go, favoring reduced hours, job shifting, and other tricks to keep people engaged and employed for the hopefully short time we are underutilizing them. Of course, the biggest difference between those of us in our own practices and those in employed positions is that we pay ourselves last-or not at all for the next month or two-a situation that may be the most surprising and disorienting element of this outbreak. In the community, the need for reliable neurosurgeons may wax or wane slightly depending on geography, but in general, there is always work, and a paycheck. For the first time, across the entire country, in a way not seen even in 2008 or after 9/11, physicians in private practice are feeling like small business owners everywhere, hoping the end of this moment comes soon, but with the knowledge that regardless of the duration, our patients still need the best care we can possibly deliver.

https://thejns.org/doi/abs/10.3171/2020.4.JNS201033

\section{Disclosures}

The authors report no conflict of interest.

\section{Correspondence}

J. Adair Prall: ap1964@comcast.net.

INCLUDE WHEN CITING

Published online April 24, 2020; DOI: 10.3171/2020.4.JNS201033. 